\title{
Predictors of Effect of Ampicillin-Sulbactam against TEM-1 $\beta$-Lactamase-Producing Escherichia coli in an In Vitro Dynamic Model: Enzyme Activity versus MIC
}

\author{
ALEXANDER A. FIRSOV,${ }^{1}$ DANIELE SAVARINO, ${ }^{2}$ MATTHEW RUBLE ${ }^{3}$ DEBORAH GILBERT, ${ }^{3}$ \\ BRENDA MANZANO, ${ }^{2}$ ANTONE A. MEDEIROS, ${ }^{2}$ AND STEPHEN H. ZINNER ${ }^{3 *}$ \\ Division of Infectious Diseases, Department of Medicine, Brown University, Roger Williams Medical Center and Rhode \\ Island Hospital, Providence, Rhode Island 02908, ${ }^{3}$ Miriam Hospital, Providence, Rhode Island 02906, ${ }^{2}$ \\ and Department of Pharmacokinetics, Institute of Biotechnology, Moscow, Russia ${ }^{1}$
}

Received 7 April 1995/Returned for modification 3 August 1995/Accepted 30 November 1995

\begin{abstract}
The clinical outcome in patients treated with ampicillin-sulbactam may not always be predictable by disc susceptibility testing or with the MIC as determined with a constant level $(4 \mu \mathrm{g} / \mathrm{ml})$ of the $\beta$-lactamase inhibitor $\left(\right.$ MIC $\left._{1}\right)$. The enzyme activities (EA) and the MICs estimated at a constant ratio of ampicillin to sulbactam of 2:1 $\left(\mathrm{MIC}_{2}\right)$ for 15 TEM-1 $\beta$-lactamase-producing strains of Escherichia coli were examined as alternatives to $\mathrm{MIC}_{1}$ as predictors of the antibacterial effects of this combined drug as studied in an in vitro model which simulates ampicillin-sulbactam pharmacokinetic profiles observed in human peripheral tissues. Integral parameters describing the area under the bacterial count-time curve (AUBC), the area between the normal growth curve, and the killing curve of bacteria exposed to antibiotic (ABBC), and the second parameter expressed as a percentage of its maximal hypothetical value $\left(\mathrm{ABBC} / \mathrm{ABBC} \mathrm{max}_{\max }\right)$ were calculated. All three parameters correlated well with $\mathrm{EA}\left(\mathrm{AUBC}, r=0.93\right.$; $\left.\mathrm{ABBC}, r=-0.88 ; \mathrm{ABBC}^{-\mathrm{ABBC}} \mathrm{max}_{\mathrm{max}}, r=-0.91\right)$ and with $\mathrm{MIC}_{2}\left(r=0.94,-0.94\right.$, and -0.95 , respectively) but not with $\mathrm{MIC}_{1}$. Both EA and $\mathrm{MIC}_{2}$ can be considered reliable predictors of the antibacterial effect of ampicillin-sulbactam in an in vitro model. These correlations suggest that in vitro kinetic-dynamic models might be useful to reexamine established susceptibility breakpoints obtained with data based on the MIC $_{1}$ (MICs obtained with constant levels of $\beta$-lactamase inhibitors). These data also suggest that quantitative determinations of bacterial $\beta$-lactamase production and MICs based on the component concentration ratio observed in vivo might be useful predictors of the effect of ampicillinsulbactam and other $\beta$-lactam-inhibitor combinations.
\end{abstract}

The search for optimal in vitro determinants or predictors of clinical outcome for antibiotics and of the antibacterial effect itself remains important in antimicrobial chemotherapy. Serious problems are associated with documenting the relation between the effect and its predictors, such as (i) the presence of numerous patient variables that mask the relation, (ii) difficulties in quantitating antibacterial and clinical effects, and (iii) insufficiently precise estimates of intrinsic antibiotic activity in terms of MICs or MBCs, which also do not consider the pharmacokinetics of the antibiotics tested (4). Nonetheless, in vitro antibacterial tests including MIC determinations are widely used to select an appropriate drug in general practice.

With ampicillin-sulbactam, clinical results are not always predicted by the MIC, especially when a constant level of the $\beta$-lactamase inhibitor is used; clinical cures of documented infections by using ampicillin plus sulbactam have occurred in the face of resistant or intermediate susceptibility results (10). We have reported similar observations using an in vitro dynamic model (3a). In vitro simulation of human pharmacokinetics of ampicillin-sulbactam produced almost identical bacterial killing kinetics for Escherichia coli strains for which the MICs varied widely ( 8 to $>1,000 \mu \mathrm{g} / \mathrm{ml}$ ), as determined with a constant level of sulbactam $(4 \mu \mathrm{g} / \mathrm{ml})$. Therefore, it is reasonable to search for alternative predictors of the antibacterial effect of this $\beta$-lactam-inhibitor combination.

In the present study, quantitative determination of $\beta$-lacta-

* Corresponding author. Mailing address: Department of Medicine, Roger Williams Medical Center, 825 Chalkstone Ave., Providence, RI, 02908. Phone: (401) 456-2074. Fax: (401) 456-2016. mase activity has been examined as a potential predictor of ampicillin-sulbactam antibacterial effect against TEM-1 $\beta$-lactamase-producing E. coli strains. The antibacterial effect was evaluated in an in vitro dynamic model simulating single-dose human pharmacokinetics of the drug.

\section{MATERIALS AND METHODS}

In vitro model, operating procedure, and simulated pharmacokinetic profiles. An in vitro pharmacokinetic-pharmacodynamic model described previously was used to simulate human tissue antibiotic profiles achieved with single intravenous doses of ampicillin-sulbactam $(2,3)$. Briefly, this model consists of a sterile central compartment representing the systemic circulation (which includes a central reservoir, tubing, and the lumina of capillary bundles) and a peripheral compartment which represents extravascular sites of infection and which includes six artificial hollow fiber capillary units (Vitafiber; Amicon Corp., Lexington, Mass.) placed in series $(2,3)$. Bacteria are placed in the peripheral compartment chambers, and antibiotics are injected into the central compartment and pumped to the peripheral chambers, where they diffuse through the selectively permeable capillary walls (with a molecular mass cutoff of $10,000 \mathrm{Da}$ ) into the units containing the bacteria. Bacteria do not penetrate into the central compartment.

The system was filled with sterile Mueller-Hinton broth supplemented with calcium $(50 \mu \mathrm{g} / \mathrm{ml})$ and magnesium $(25 \mu \mathrm{g} / \mathrm{ml})$ and was placed in an incubator at $37^{\circ} \mathrm{C}$. The peripheral compartments were inoculated through rubber septa $2 \mathrm{~h}$ before antibiotics were added, and the resulting exponentially growing cultures approximated $10^{4} \mathrm{CFU} / \mathrm{ml}$. Peristaltic pumps circulated antibiotic-containing media from the central reservoir through the tubing and the capillary lumina and finally back to the central reservoir at a rate of $3 \mathrm{ml} / \mathrm{min}$. The contents of the peripheral chambers containing $10 \mathrm{ml}$ of broth and bacteria plus antibiotics also were circulated by peristaltic pumps.

Broth in the central compartment was continuously eliminated and replaced with fresh medium from a diluent reservoir to mimic the drug concentration-time profiles in peripheral tissue as observed in humans following single-dose intravenous administration of ampicillin-sulbactam $(2 \mathrm{~g} / 1 \mathrm{~g})$. In all experiments, monoexponential concentration decays were simulated with an elimination half- 
life of $1 \mathrm{~h}$ (11). Control growth in the absence of antibiotics was studied in the same model.

Ampicillin concentrations in the central and peripheral compartments were determined by bioassays. Antibiotic medium no. 1 (Difco) seeded with Bacillus subtilis ATCC 6633 (Difco-Subtilis Spore Suspension) was used to prepare bioassay plates. Wells were made in the agar and were filled with $25 \mu \mathrm{l}$ of sample. The plates were incubated overnight at $37^{\circ} \mathrm{C}$. After overnight incubation, the zone sizes were measured by using calipers. Ampicillin concentrations were calculated against a four-point standard curve by linear regression. The observed concentrations were close to the designed values, with no systematic deviation from expected values. At concentrations of between 4.7 to $75 \mu \mathrm{g} / \mathrm{ml}$, sulbactam did not interfere with ampicillin determinations.

Bacterial strains. Fourteen strains of $E$. coli that produced only TEM-1 $\beta$-lactamase as determined by isoelectric focusing (14) were tested. Most were resistant to ampicillin (MIC, $>1,000 \mu \mathrm{g} / \mathrm{ml}$ ). One strain (E. coli 93-4300) did not produce $\beta$-lactamase (MIC, $7.8 \mu \mathrm{g} / \mathrm{ml}$ ). All were clinical isolates.

EA determinations. The $\beta$-lactamase activities (enzyme activity [EA], expressed as micromoles of cephaloridine [ $100 \mu \mathrm{mol}$ used as substrate] hydrolyzed per minute per milligram of protein) were determined spectrophotometrically (12). An average of five determinations were performed for each strain.

Determinations of MICs. MICs were determined by the broth microdilution technique, with inocula of approximately $5 \times 10^{5} \mathrm{CFU} / \mathrm{ml}$ (9) under two different conditions regarding the ratio of ampicillin to sulbactam. The bacteria were stored in liquid nitrogen and were preincubated at $37^{\circ} \mathrm{C}$ for 2 to $5 \mathrm{~h}$ to achieve the desired inoculum.

Method 1. MICs as determined with a constant level of the $\beta$-lactamase inhibitor $\left(\mathrm{MIC}_{1} \mathrm{~s}\right)$ were estimated at different ampicillin-to-sulbactam ratios by varying the ampicillin concentrations with a constant concentration of sulbactam of $4 \mu \mathrm{g} / \mathrm{ml}$.

Method 2. MICs estimated by using a constant (2:1) ratio of ampicillin to sulbactam $\left(\mathrm{MIC}_{2} \mathrm{~s}\right)$ were determined by varying the ampicillin and $\beta$-lactamase inhibitor concentrations in parallel.

Quantitation of bacterial growth and killing. In each experiment, 0.3-ml samples were withdrawn from the peripheral compartments seven to eight times during the 8-h period which reflects the usual dosing interval in patients treated with ampicillin-sulbactam. These samples were subjected to serial 10 -fold dilutions with chilled, sterile $0.9 \% \mathrm{NaCl}$ and were plated in triplicate on MuellerHinton agar. In addition, $100 \mu \mathrm{l}$ of each sample was filtered through a $0.45-\mu \mathrm{m}-$ pore-size membrane filter (HA [47-mm diameter]; Millipore Corp., Bedford, Mass.) and was also plated on agar. After incubation at $37^{\circ} \mathrm{C}$, the resulting bacterial colonies were counted and the numbers of CFU per milliliter were calculated. Incubation times varied from 16 to $48 \mathrm{~h}$, depending on the strain and plating method (direct or filter). With this model, most treated strains were studied in duplicate or triplicate. Two untreated strains were tested in duplicate. The standard deviation of the bacterial counts (both for treated and untreated bacteria) of replicate samples averaged $0.32 \log$ CFU.

Quantitative evaluation of antibacterial effect. To compare bacterial killing among strains, an integral parameter was used to reflect the area under the bacterial count-time curve (AUBC) $(7,13,15)$. On the basis of the bacterial count-time data obtained in the presence of ampicillin-sulbactam, trapezoidal areas under the log CFU per milliliter-time curves for the 8-h observation period were calculated. The wide hatched area under this curve (Fig. 1; the lower border of the closely hatched area) includes initial bacterial killing and regrowth and is a unique profile of changes in bacterial counts for a given organism under any particular dose and regimen. Not only to express these changes, i.e., bacterial count dynamics in the presence of a drug, but also to reflect the actual antibacterial effect, the difference between the AUBC in the absence of antibiotics $\left(A \mathrm{ABC}_{\text {control }}\right)$ and the AUBC in the presence of the drug was estimated (the area between the bacterial growth curves $[\mathrm{ABBC}]$; see the closely hatched area in Fig. 1).

ABBC may be considered another estimate of a previously described integral parameter representing the intensity of the antibacterial effect, $\mathrm{I}_{\mathrm{E}}$-area between bacterial killing-regrowth and normal growth curves up to the point when bacterial counts in the regrowth curve reach the maximal values observed in the absence of antibiotics (6). However, unlike this parameter, ABBC can be determined within a fixed observation period regardless of the phase of the bacterial kinetic curve observed in the presence of antibiotics. ABBC results are expressed in (log CFU per milliliter) $\times$ hours. To express the ampicillin-sulbactam antibacterial effect as a percentage of the hypothetical maximum value of $\mathrm{ABBC}$ (i.e., $\mathrm{ABBC}_{\max }=\mathrm{AUBC}_{\text {control }}$ ), the ratios of $\mathrm{ABBC}$ to $\mathrm{ABBC}_{\max }$ were calculated.

Stepwise multiple correlation and regression analyses of the relations between the $\mathrm{AUBC}, \mathrm{ABBC}$, and $\mathrm{ABBC} / \mathrm{ABBC}_{\max }$ and the MICs or EA as well as those between MICs and EA were performed with STATGRAF software (STSC, Inc.).

\section{RESULTS}

The ampicillin-sulbactam combination produced pronounced reductions in the inocula for most of the 15 strains studied. However, as seen in the sample curves (Fig. 2), bacterial regrowth occurred in different patterns by the end of the

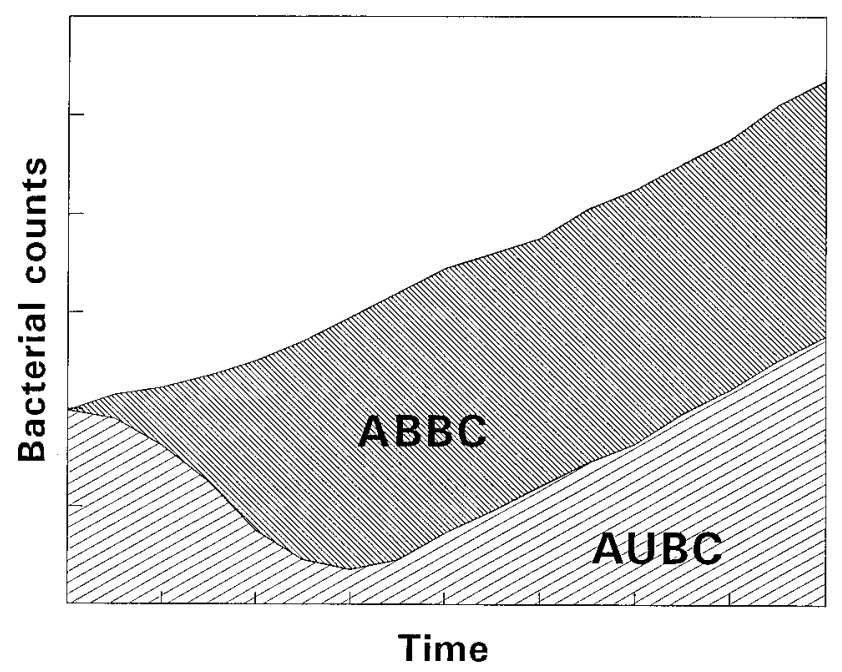

FIG. 1. Determination of the ABBC as the area between the normal growth curve (the upper border of the closely hatched area) and the curve of bacteria exposed to drugs (the bottom border of the closely hatched area). The AUBC is reflected by the widely hatched area under the killing curve.

8-h observation period with 13 of the 15 E. coli strains. The two strains that did not regrow are not represented in Fig. 2. As seen in Table 1 , the differences in bacterial killing curves observed with the 15 strains studied in this model could not be explained by differences in $\mathrm{MIC}_{1} \mathrm{~s}$ determined at the constant concentration of sulbactam (method 1). Moreover, since most of the $\mathrm{MIC}_{1} \mathrm{~s}$ exceeded 1,000 $\mu \mathrm{g} / \mathrm{ml}$ (Table 1), it was impossible to more accurately correlate the antibacterial effect with $\mathrm{MIC}_{1}$.

At the same time, the obvious differences in the killing curves (Fig. 2) for three E. coli strains for which the $\mathrm{MIC}_{1} \mathrm{~s}$ were similar but for which the EA were different could be explained by differences in the EA but not in the $\mathrm{MIC}_{1}$. In addition, $\mathrm{AUBC}, \mathrm{ABBC}$, or $\mathrm{ABBC} / \mathrm{ABBC}_{\max }$ did not correlate with $\mathrm{MIC}_{1}$ (data not shown).

However, EA values were distinctly correlated with AUBC, $\mathrm{ABBC}$, and $\mathrm{ABBC} / \mathrm{ABBC}_{\max }$ values (Fig. 3). The plot of AUBC versus EA (Fig. 3) shows that higher enzyme produc-

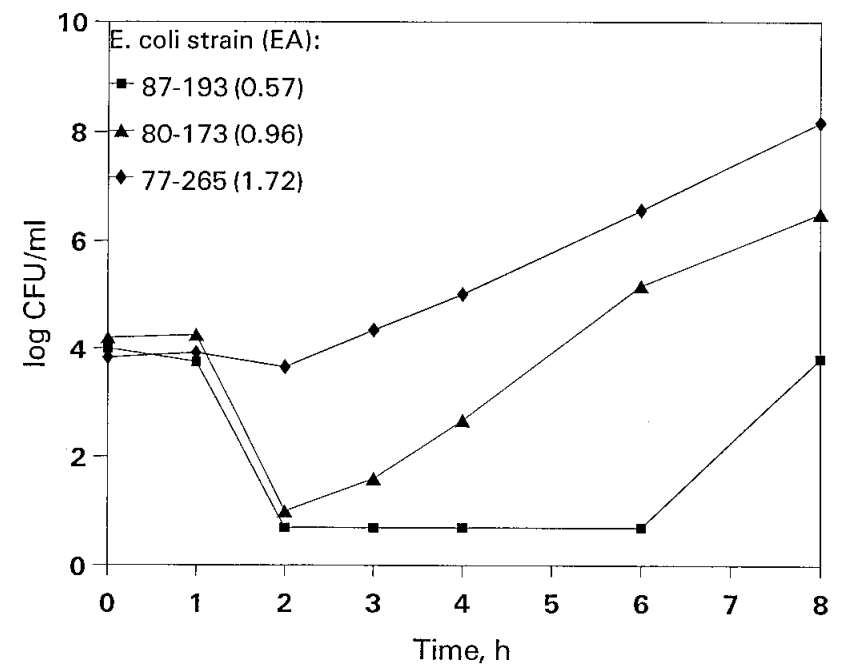

FIG. 2. Ampicillin-sulbactam-induced killing curves obtained with three $E$. coli strains for which the $\mathrm{MIC}_{1} \mathrm{~s}$ are $>1,000 \mu \mathrm{g} / \mathrm{ml}$ and for which the EA differ. 
TABLE 1. EA, $\mathrm{MIC}_{1} \mathrm{~s}$, and $\mathrm{MIC}_{2} \mathrm{~s}$ of $E$. coli strains

\begin{tabular}{cccc}
\hline E. coli strain & $\mathrm{EA}(\mu \mathrm{mol} / \mathrm{min} / \mathrm{mg})$ & $\mathrm{MIC}_{1}(\mu \mathrm{g} / \mathrm{ml})^{a}$ & $\mathrm{MIC}_{2}(\mu \mathrm{g} / \mathrm{ml})^{a}$ \\
\hline $77-265$ & $1.72 \pm 0.11$ & $>1,000$ & 250.0 \\
$78-419$ & $1.64 \pm 0.07$ & $>1,000$ & 125.0 \\
$80-173$ & $0.96 \pm 0.07$ & $>1,000$ & 62.5 \\
$80-354$ & $1.57 \pm 0.03$ & $>1,000$ & 250.0 \\
$82-320$ & $1.62 \pm 0.08$ & $>1,000$ & 250.0 \\
$82-435$ & $0.26 \pm 0.02$ & $>1,000$ & 62.5 \\
$86-460$ & $0.70 \pm 0.06$ & 1,000 & 44.2 \\
$87-140$ & $0.15 \pm 0.03$ & 22.1 & 15.6 \\
$87-193$ & $0.57 \pm 0.02$ & $>1,000$ & 31.3 \\
$88-019$ & $0.76 \pm 0.08$ & $>1,000$ & 125.0 \\
$88-436$ & $0.70 \pm 0.06$ & $>1,000$ & 88.4 \\
$88-455$ & $0.63 \pm 0.06$ & $>1,000$ & 44.2 \\
$89-300$ & $0.18 \pm 0.02$ & 11.0 & 11.0 \\
$91-1151$ & $0.86 \pm 0.07$ & $>1,000$ & 125.0 \\
$93-4300$ & 0.00 & 3.9 & 3.9 \\
\hline
\end{tabular}

${ }^{a}$ Geometric means of as many as five determinations.

tion is associated with a larger AUBC, i.e., a smaller antibacterial effect. Thus, bacteria which produce a large amount of $\beta$-lactamase are killed less readily than those which produce smaller amounts of the enzyme. The antibacterial effect is therefore inversely proportional to EA, since the AUBC is directly proportional to the EA. Since the ABBC is inversely proportional to EA (higher enzyme activity resulting in less antibacterial effect), this parameter might be inherently more logical because a higher enzyme activity would be expected to correlate with less bacterial killing. Similarly, the relation of the $\mathrm{ABBC} / \mathrm{ABBC} \mathrm{max}_{\text {max }}$ to the $\mathrm{EA}$ is also consistent with expected inverse proportionality to the EA. The latter relation is the most convenient, since it provides the prediction of the antibacterial effect in terms of a percentage of its maximal value.

These data suggest that EA determination might be a better in vitro predictor of antibacterial effect than the $\mathrm{MIC}_{1}$ determined at a constant concentration of sulbactam, i.e., at an arbitrary ampicillin-sulbactam ratio. Unlike the EA, the $\mathrm{MIC}_{1}$ did not correlate with any of the antibacterial-effect parameters. However, when $\mathrm{MIC}_{2} \mathrm{~s}$ (with a constant ratio of ampicillin to sulbactam of 2:1 [method 2]) were studied, good correlations were found with the $\mathrm{MIC}_{2} \mathrm{~s}$ and the AUBC, ABBC, and $\mathrm{ABBC}_{\mathrm{ABBC}}$ max values (Fig. 4). Therefore, $\mathrm{MIC}_{2}$ (with a constant 2:1 ratio) but not $\mathrm{MIC}_{1}$ (with a constant sulbactam concentration) is an acceptable predictor of the antibacterial effect of ampicillin-sulbactam.

The presence of an interrelation of two in vitro predictors might verify the observations shown here with the new parameters $\mathrm{AUBC}, \mathrm{ABBC}$, and $\mathrm{ABBC} / \mathrm{ABBC}_{\max }$. The strong correlation between EA and $\mathrm{MIC}_{2}$ (Fig. 5) is consistent with their respective correlations with the antibacterial-effect parameters. Because of the strong interrelation between EA and $\mathrm{MIC}_{2}$, simultaneous use of both predictors in the matrix of stepwise multiple correlation analysis did not result in moreaccurate prediction of the $\mathrm{AUBC}, \mathrm{ABBC}$, and $\mathrm{ABBC} / \mathrm{ABBC}_{\text {max }}$ values; the multiple correlation coefficients were insignificantly higher than the respective paired correlation coefficients.

\section{DISCUSSION}

The optimal role of in vitro determinations of antibacterial activity in predicting clinical results remains unclear. Most clinical microbiology laboratories report susceptibility data in terms of MICs of antibiotics reflecting actual MIC determinations or surrogate disc size measurements. With some antibiotics, especially ampicillin-sulbactam and possibly other $\beta$-lac-
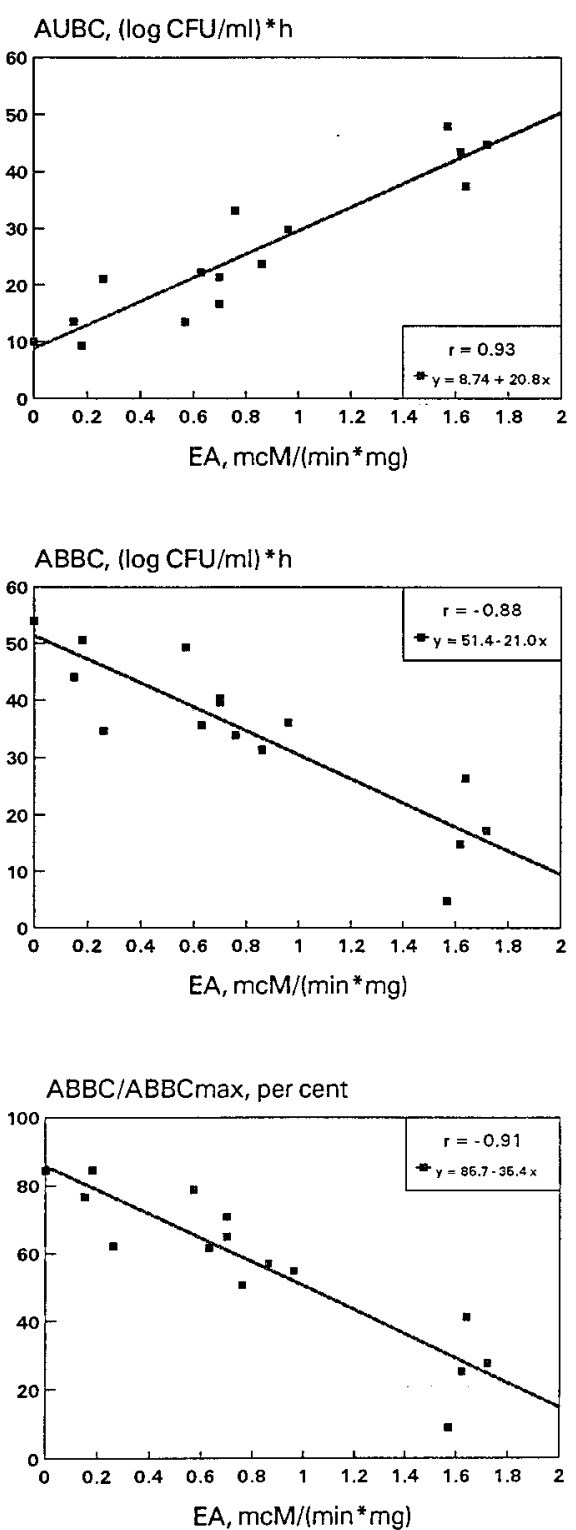

FIG. 3. Correlations between integral parameters of the effects of ampicillinsulbactam on 15 strains of $E$. coli and EA

tam- $\beta$-lactamase inhibitor combinations, the clinical results may exceed those predicted by routine in vitro sensitivity data (10). Thus, there is a need to search for alternative predictors or determinants of antibacterial effect and clinical outcome.

Although clinical data are ultimately necessary to determine the value of in vitro predictors, it is often difficult to discriminate or select among these predictors (4). To make the discrimination more accurate, bacterial-killing kinetic data obtained with in vitro simulations of human pharmacokinetic profiles are used as an alternative basis for the predictor selection $(1,16)$. This approach was applied in our investigation to discriminate between a traditional predictor (MIC) and a new predictor (EA) of the antibacterial effect of ampicillinsulbactam. Bacterial killing following exposure to simulated single-dose intravenous administration of ampicillin-sulbactam was studied in the dynamic model.

Good correlations were found with several new estimates of 
AUBC, $(\log \mathrm{CFU} / \mathrm{ml}) * \mathrm{~h}$

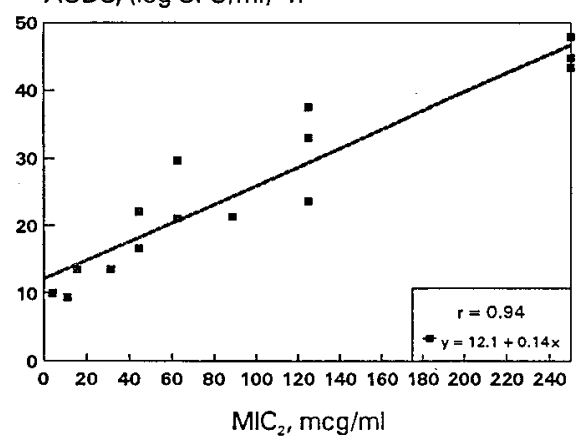

ABBC, $(\log \mathrm{CFU} / \mathrm{ml}) * \mathrm{~h}$

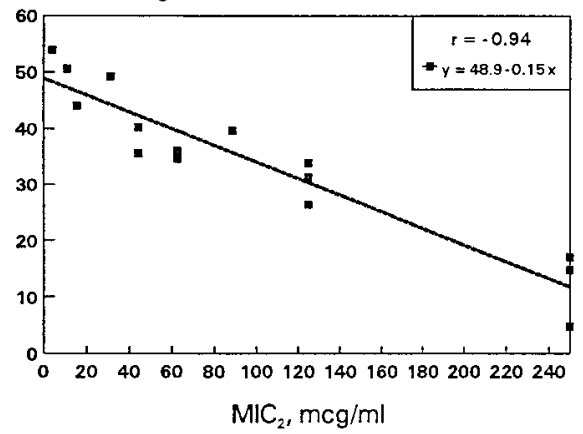

$A B B C / A B B C$ max, per cent

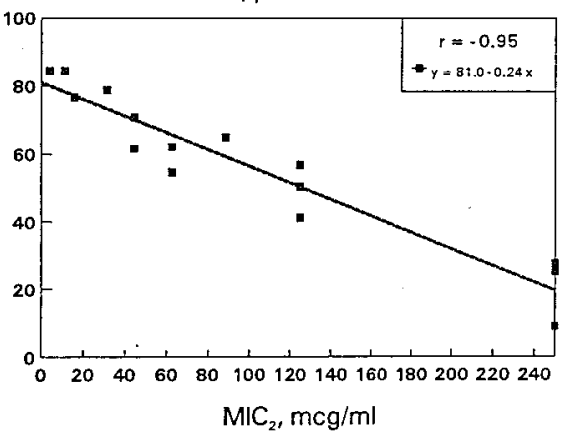

FIG. 4. Correlations between integral parameters of the effects of ampicillinsulbactam on 15 strains of $E$. coli and $\mathrm{MIC}_{2} \mathrm{~s}$.

antibacterial effect (AUBC, $\mathrm{ABBC}$, and $\mathrm{ABBC} / \mathrm{ABBC}_{\text {max }}$ ) and EA of TEM-1 $\beta$-lactamase-producing $E$. coli, but not with the MICs as determined at a fixed concentration of sulbactam $\left(\mathrm{MIC}_{1}\right)$. This observation is not surprising, for in most cases the $\mathrm{MIC}_{1} \mathrm{~s}$ were obtained at ratios of ampicillin to sulbactam which differed from ratios observed in humans and those simulated in the dynamic model, as well as the 2:1 component ratio in the drug itself. For example, when $\mathrm{MIC}_{1}$ was equal to $>1,000 \mu \mathrm{g} / \mathrm{ml}$, it reflected the activity of ampicillin-sulbactam at the concentration ratio of $>250: 1$, i.e., at conditions of pronounced deficiency of the inhibitor. It has been shown previously that the antibacterial effect of ampicillin-sulbactam is extremely sensitive to the ratio of these components, especially when that ratio exceeds $2: 1$ (8). It is certainly possible that $\mathrm{MIC}_{1}$ and $\mathrm{MIC}_{2}$ would have correlated well with the new parameters and with each other if only very susceptible strains had been studied.

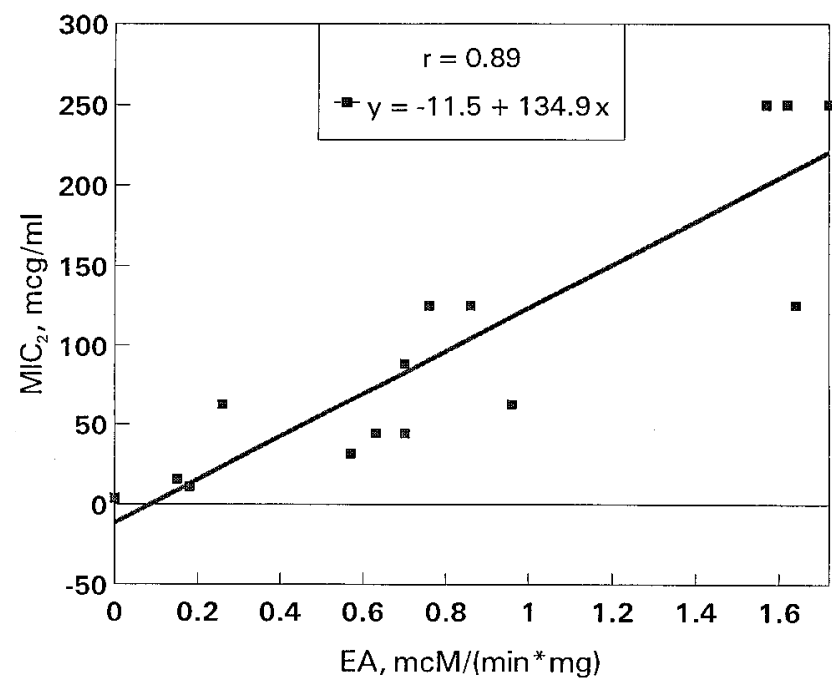

FIG. 5. Correlation between $\mathrm{MIC}_{2} \mathrm{~s}$ and EA for 15 strains of E. coli.

That good correlations with the antibacterial-effect parameters and MICs determined at a constant ratio (2:1) of ampicillin to sulbactam $\left(\mathrm{MIC}_{2}\right)$ but not with the $\mathrm{MIC}_{1}$ were found in this study also demonstrates the importance of the antibiotic-inhibitor ratio. Thus, the data presented suggest that both EA and $\mathrm{MIC}_{2}$ are likely to be useful in reexamining established susceptibility breakpoints based on data obtained with fixed concentrations of $\beta$-lactamase inhibitors.

The in vitro kinetic-dynamic model used here provided a unique ability to study the kinetics of antibacterial effects by simulating human pharmacokinetic profiles. In addition, three parameters to quantitatively evaluate antibacterial effects were described and tested. All of these are integral parameters and provide an unequivocal evaluation of ampicillin-sulbactam's antibacterial effect in each experiment. A similar approach has been validated recently (6) and has been applied to in vitro simulation of the pharmacokinetic profiles of cefotaxime and sisomicin (5).

Although the three integral parameters described are of similar natures, clearly covary, and provide equally good correlations with bacterial $\beta$-lactamase activity as well as with $\mathrm{MIC}_{2}$, the correlations obtained with $\mathrm{ABBC}$ or $\mathrm{ABBC} / \mathrm{ABBC}_{\max }$ are preferable to those obtained with $\mathrm{AUBC}$, since the first two parameters consider the intrinsic features of each strain in terms of its growth pattern in the absence of antibiotics. Unlike the AUBC or the area above the curve described by Wiedemann and Jansen (17), the algebraic sum of the areas between the bacterial killing curve and the line representing the initial inoculum, the $\mathrm{ABBC}$, and the $\mathrm{ABBC} / \mathrm{ABBC}_{\text {max }}$ relate antibiotic-induced bacterial killing to the control growth of each individual strain. ABBC might be a direct measure of the antimicrobial effect.

Overall, these results suggest that $\beta$-lactamase production by a given bacterial strain and the MIC as determined with a fixed ratio of ampicillin to sulbactam that reflects their concentration ratios in humans and in the drug formulation might be better in vitro predictors of antibacterial action than the standard MIC determined with a fixed concentration of sulbactam. Obviously, clinical correlations are needed. Furthermore, in vitro pharmacokinetic-pharmacodynamic models that incorporate pharmacokinetic parameters might be useful tools to search for and discriminate among predictors of antibacterial effects. 


\section{ACKNOWLEDGMENT}

This study was supported by Roerig, a division of Pfizer Pharmaceuticals.

\section{REFERENCES}

1. Bastone, E. B., S. C. Li, L. L. Ioannides-Demos, W. J. Spicer, and A. J. McLean. 1993. Kill kinetics and regrowth pattern of Escherichia coli exposed to gentamicin concentration-time profiles simulating in vivo bolus and infusion dosing. Antimicrob. Agents Chemother. 37:914-917.

2. Blaser, J., B. B. Stone, and S. H. Zinner. 1985. Two compartment kinetic model with multiple artificial capillary units. J. Antimicrob. Chemother. 15(Suppl. A):131-137.

3. Blaser, J., B. B. Stone, and S. H. Zinner. 1985. Efficacy of intermittent versus continuous administration of netilmicin in a two-compartment in vitro model. Antimicrob. Agents Chemother. 27:343-349.

3a.Firsov, A., D. Saverino, M. Ruble, D. Gilbert, B. Manzano, A. Medeiros, and S. Zinner. 1993. The relation of ampicillin/sulbactam (A/S) action to bacterial beta-lactamase production in an in vitro dynamic model. Program Abstr. 33rd Intersci. Conf. Antimicrob. Agents Chemother., abstr. 273. American Society for Microbiology, Washington, D.C.

4. Firsov, A. A. 1993. Critical reappraisal of modern approaches to search determinants of efficacy of antimicrobials. 1993. Eur. Bull. Drug Res. 2(Suppl. 1):33-38.

5. Firsov, A. A. 1991. In vitro simulated pharmacokinetic profiles: forecasting antibiotic optimal dosage. Eur. J. Drug Metab. Pharmacokinet. (Special Issue) 3:406-409.

6. Firsov, A. A., V. M. Chernykh, and S. M. Navashin. 1991. Quantitative analysis of antimicrobial effect kinetics in an in vitro dynamic model. Antimicrob. Agents Chemother. 34:1312-1317.

7. Firsov, A. A., A. D. Nazarov, and V. M. Chernykh. 1989. Pharmaco-kinetic approaches to rational antibiotic therapy. In Advances in science and engineering, vol. 17. VINITI Publishers, Moscow. (In Russian.)

8. Firsov, A. A., A. D. Nazarov, V. M. Chernykh, and S. M. Navashin. 1988.
Validation of optimal ampicillin/sulbactam ratio in dosage forms using invitro dynamic model. Drug Dev. Ind. Pharm. 14:2425-2442.

9. Gavan, T. L., and A. L. Barry. 1980. Microdilution test procedures, p. 459-462. In E. H. Linnette (ed.), Manual of clinical microbiology, 3rd ed. American Society for Microbiology, Washington, D.C.

10. Jochimsen, W. G., D. J. Sheehan, and G. Mylott. 1995. Ampicillin/sulbactam $(\mathrm{A} / \mathrm{S})$ : correlation between in vitro antimicrobial susceptibility testing and clinical activity, abstr. C-114, p. 20. Abstr. 95th Annu. Meet. Am. Soc. Microbiol. 1995. American Society for Microbiology, Washington, D.C.

11. Kinishi, T. 1993. Pharmacokinetic profile of antimicrobial agents. II-1. Penicillins, p. 43-64. In H.-P. Kuemmerle, T. Murakawa, and C. H. Nightingale (ed.), Pharmacokinetics of antimicrobial agents: principles, methods, application. Ecomed Verlag, Landsberg, Germany.

12. O'Callaghan, C. H., A. Morris, S. M. Kirby, and A. H. Shingler. 1972. Novel method for detection of beta-lactamase by using a chromogenic cephalosporin substrate. Antimicrob. Agents Chemother. 1:283-288.

13. Tisdale, J. E., M. T. Pasko, and J. M. Mylotte. 1989. Antipseudomonal activity of simulated infusions of gentamicin alone or with piperacillin assessed by serum bactericidal rate and area under the killing curve. Antimicrob. Agents Chemother. 33:1500-1505.

14. Vecoli, C., F. E. Prevost, J. J. Ververis, A. A. Medeiros, and G. P. O'Leary, Jr. 1983. Comparison of polyacrylamide and agarose gel thin-layer isoelectric focusing for the characterization of $\beta$-lactamases. Antimicrob. Agents Chemother. 24:186-189.

15. White, C. A., and R. D. Toothaker. 1985. Influence of ampicillin elimination half-life on in vitro bactericidal effect. J. Antimicrob. Chemother. 15(Suppl. A):257-260.

16. White, C. A., R. D. Toothaker, A. L. Smith, and J. T. Slattery. 1989. In vitro evaluation of the determinants of bactericidal activity of ampicillin dosing regimens against Escherichia coli. Antimicrob. Agents Chemother. 33:10461051.

17. Wiedemann, B., and A. Jansen. 1990. Antibacterial activity of cefpodoxime proxetil in a pharmacokinetic in vitro model. J. Antimicrob. Chemother. 26:71-79. 